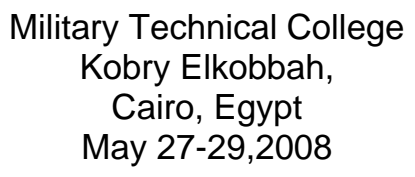

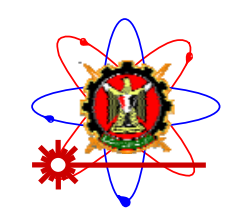
$4^{\text {th }}$ International Conference on Mathematics and Engineering Physics (ICMEP-4)

EM-18

\title{
An Optimization Approach for Aircraft Sparing
}

\author{
Atef Ayed Ayed, Elsayed Sorour, ADLY Istafanos, Fawzi Ibrahim
}

\begin{abstract}
The objective of this paper is to establish an optimum recommended spare list of aircraft components. The criteria of selecting a spare item for purchase are based on its ability to improve the aircraft operational availability. Marginal analysis is used to sort the spares such that optimum budget utilization is achieved. A mathematical model for aircraft operational availability is derived through the analysis of a typical aircraft operation and maintenance concept. A complex queuing network of aircraft operation and maintenance is simplified to help in deriving the mathematical model for aircraft operational availability. This approach is developed to support the decision making process during the provisioning of aircraft spares. A typical example and its result analysis are given. Also, a complete user-friendly package is developed and implemented using Visual Basic 6.
\end{abstract}

\section{KEY WORDS}

Aircraft, operational availability, spares, spare list, components, operation and maintenance, optimum budget and mathematical model.

\section{NOMENCLATURE}

$\mathrm{A}_{0} \quad$ Operational availability

C Cost of an item

DDR Daily demand rate

DFH Daily flying hours

EBO Expected back order

I Improvement factor

MTBD Mean time between demand

MTTR Mean time to repair

NAC Number of aircraft

NRTS Not repair this station ratio

PL Pipe line

q Item operational availability

QPA Quantity per application

$\mathrm{S} \quad$ Stock level of item an item

SF Sort factor

TTRD Time to repair in the depot level

TTRD Time to repair at depot maintenance level

TTRI Time to repair in the intermediate level

$\mathrm{X} \quad$ Number of items in the resupply 


\section{INTRODUCTION}

Over the last few years, one can see the tremendous achievement made in technology. Systems in the future will be technologically more advanced than those of today will. While technology is advanced at a rapid rate, the complexity of the aircraft system increases at the same time too [1]. This rapid change in technology influences the operational availability $\left(\mathrm{A}_{0}\right)$, cost, and etc. In this work, a study of an aircraft system and its $\mathrm{A}_{0}$ is presented. In addition, a mathematical model is used to compute the $\mathrm{A}_{0}$ of an aircraft system in use as soon as to compare between different aircraft designs. To implement the mathematical model, a specified computer program is required. This program is very user friendly and can be helpful in the decision making process as a decision making tool. The objective of this work is to establish an optimum recommended spare list of aircraft components. Therefore, the mathematical modeling of the $\mathrm{A}_{0}$ is given in section 3 . The optimum aircraft sparing is given in section 3.3. The optimization algorithm is discussed in section 3.4. A typical example is introduced in section 4. The results analysis and conclusion are given in section 6 .

\section{MATHEMATICAL MODELLING OF AIRCRAFT AVAILABILITY}

The operation and maintenance of the aircraft system is based on three levels concept. At the first level, the organizational level, aircraft maintenance, periodic checks, visual inspections, cleaning, servicing, removal and replacement of failed components, aircraft activation and deactivation are performed. The flying missions are generated to accomplish the required aircraft utilization. The second level, the intermediate level is dedicated to repair of aircraft components, calibration, and aircraft periodic inspection. The third level of maintenance, the depot level, constitutes the highest type of maintenance and supports the accomplishment of tasks beyond the capability at the intermediate level. The depot level includes the complete overhauling, rebuilding, calibration of test and support equipment, inspection and repairing of line and shop replaceable units are performed.

Actually, the operation and maintenance of aircraft is a complex process [2,3]. This process includes a lot of interacting elements and factors. Figure 1 shows a pictorial representation of complex aircraft operation and maintenance network that is based on the three level concepts. Also, depending on the availability of serviceable components an aircraft may be ready (available), down for maintenance or down awaiting for spares. In this paper, the calculation of spares requirement is based on the relation between the component availability and the aircraft availability.

Developing a mathematical model for $\mathrm{A}_{0}$ in terms of component stock level, through the analysis of the above queuing network, is a complex task and faces the following problems and considerations:

A. The arrival and service patterns of customers (missions, aircraft, and components) are generated in character.

B. A mission, as a transaction, may occupy more than one station at a time. This may take place when a partition from a mission is waiting at the lunch queue while the rest of aircraft assigned to that mission are still in the activation storage or even undergoing maintenance in the aircraft queuing system.

C. The customers of the aircraft queuing system are activated and acquire service depending on the state of customers in the mission queuing system. Also, customers of component queuing system are generated and acquire service according to the state of customers in the other two queuing systems.

D. Customers of component queuing system are, sometimes, a subset of customers of aircraft queuing system that are a subset of customers of mission queuing system. This happens when a component is installed on an aircraft and the aircraft is assigned to a mission.

E. There is a probabilistic branching of aircraft and aircraft components according to the failure encountered and the ability of repair at the organizational and intermediate levels. Based on the probability that an aircraft is ready due to component availability (i.e. not missing a component), $\mathrm{A}_{0}$ calculation may be carried out. In this case the interest will be to determine the probability of routing an aircraft from station \#5 directly to the alert storage (station \#1). 


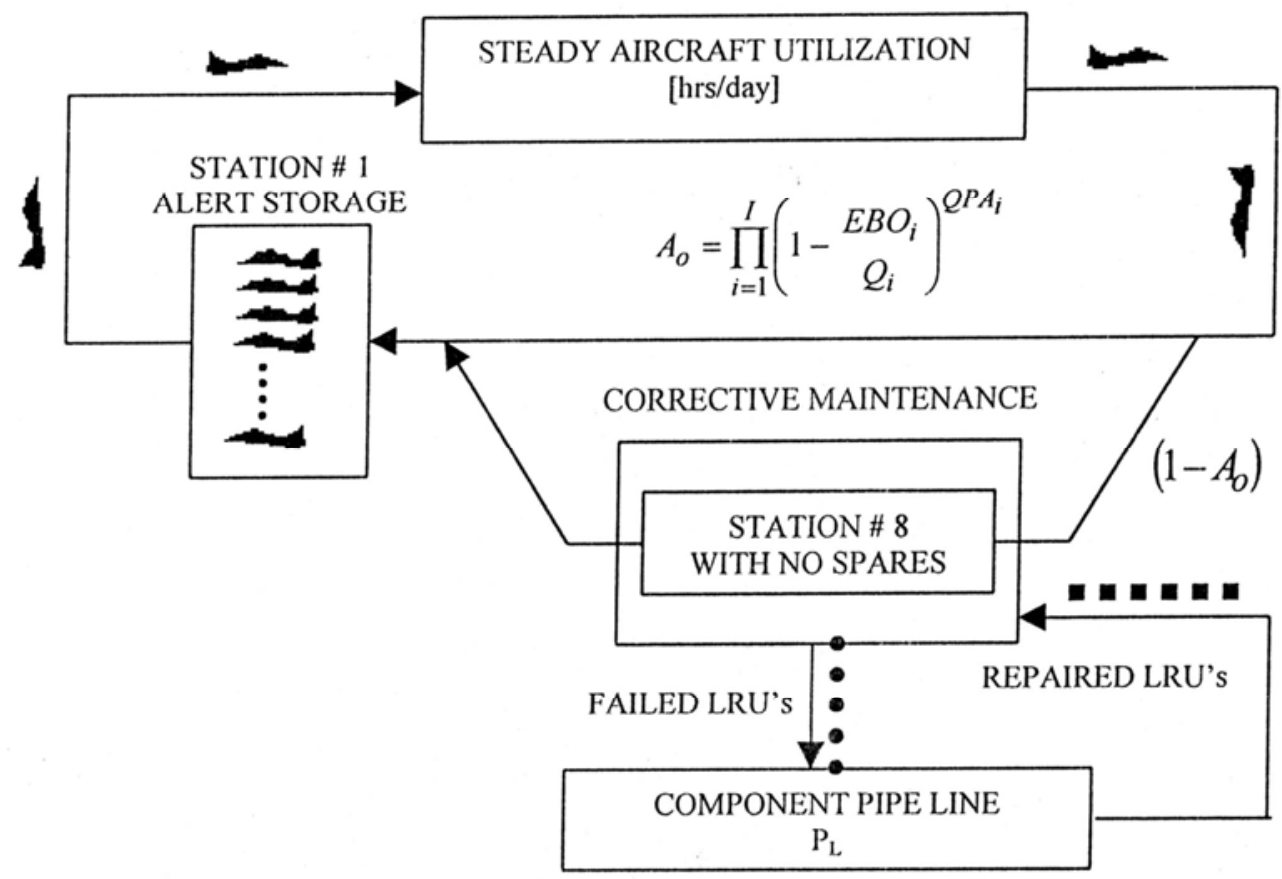

Fig. 1. Simplified aircraft queuing network

\subsection{Assumptions}

A. The effect of mission queuing system on the aircraft queuing system is substituted by steady aircraft utilization.

B. The effect of component queuing system on the aircraft queuing system is substituted by a pipeline of failed and repaired components. The quantity in the pipeline will determine the expected back order for each component for different stock levels.

C. The down time caused by deactivation, scheduled maintenance and corrective maintenance with spares is negligible compared with the down time resulted from component unavailability.

D. Tow indenture levels are considered, aircraft and aircraft components. The effect of lower levels and consumable spares is neglected.

\subsection{Calculation of Aircraft Operational Availability}

The approach for derivation an equation for the $\mathrm{A}_{0}$ in terms of component stock level and repair, is based on the belief that the major portion of aircraft downtime is caused by the component available [1]. The idea is to define $\mathrm{A}_{0}$ as a product of a series of probabilities. Each of the probabilities represents the event that a random aircraft is not missing one of its components. The more components have an aircraft the more factors are in the product.

Consider:

$$
\begin{aligned}
& E B O_{i}=\sum_{X_{i}=S_{i}+1}^{\infty}\left(X_{i}-S_{i}\right) p\left(X_{i} / P L_{i}\right) \\
& p\left(X_{i} / S_{i}\right)=\frac{P L_{i}^{S_{i}} \cdot e^{-P L_{i}}}{S_{i} !}
\end{aligned}
$$


Where:

$E B O_{i} \quad$ is the expected back order quantity of component $i$.

$i \sim 1,2, \ldots, I \quad$ I is the total number of components installed on the aircraft.

$S_{i} \quad$ is the initial stock level of component $\mathrm{i}$.

$X_{i} \quad$ is the variable stock level quantity.

$p\left(X_{i} / P L_{i}\right) \quad$ is a Poisson distribution with mean PL.

$P L_{i} \quad$ is the average pipe line quantity that can be expressed as follows:

$$
P L_{i}=D D R_{i}\left(R T S_{i} \cdot T T R I_{i}+N R T S_{i} \cdot T_{T R D_{i}}\right)
$$

Where:

$\mathrm{DDR}_{\mathrm{i}} \quad$ is the daily demand rate (item/day) of component $\mathrm{i}$. It can be expressed as follows:

$$
D D R_{i}=\frac{D F H_{i}}{M T B D_{i}}
$$

Where:

$D F H_{i} \quad$ Daily Flying Hours.

$M T B D_{i} \quad$ is the Mean Time Between Demand of component i,(hours/item).

$R T S_{i} \quad$ is the Repair This Station ratio (\%) (Station here means the intermediate level).

$N R T S_{i} \quad \mathrm{NRTSi}=(1-R T S i)$ gives the percentage of component failures that are routed to the depot level $(\%)$.

TTRI Time To Repair at the Intermediate level [days].

TTRD Time To Repair at the Depot level [days]. the

If $Q_{i}$ is considered to be the total quantity of component $i$ installed on all aircraft then

probability that a component $i$ installed on a random aircraft, is backordered is:

$E B O_{i} / Q_{i}$ 


$$
Q_{i}=N A C \cdot Q P A_{i}
$$

The probability that an aircraft is not missing a component $i$ will be:

$$
q_{i}=1-\frac{E B O_{i}}{Q_{i}}
$$

IfQPA ${ }_{i}$ is a quantity of component $i$ installed on each aircraft then:

$$
q_{i}=\left(1-\frac{E B O_{i}}{Q_{i}}\right)^{Q P A_{i}}
$$

Where:

Qi.......is the probability that a random aircraft is not waiting for a spare unit of Component $i$ with $n$ spare units of component $\mathrm{i}$ in the stock.

Assuming independence of EBO between different aircraft components, the overall $\mathrm{A}_{0}$ can be expressed as following:

$$
A_{o}=\prod_{i=1}^{I}\left(1-\frac{E B O_{i}}{Q_{i}}\right)^{Q P A_{i}}
$$

In this expression, NRTS, TTRI, TTRD, UTR, MTBD, and initial spare quantity, S, affect $\mathrm{A}_{0}$. This is due to dependence of the $E B O$ on these variables as in equation 1 through equation 8 .

\subsection{Optimum Aircraft Sparing}

The optimization policy used is a marginal analysis technique by the criteria of putting the money available in the budget in the right buy. This may be accomplished by sorting the candidate components of procurements in terms of decreasing benefit per unit cost where the benefit is defined in terms of the positive effect (increase) on the operational availability rate which would occur if that spare unit were added to the inventory [2]. For this purpose, an improvement factor of item $i$ is introduced to help in the sorting process:

$$
I_{i}=\frac{\left(q_{i}\right)_{n+1}-\left(q_{i}\right)_{n}}{\left(q_{i}\right)_{n}}
$$

Where:

$\mathrm{I}_{\mathrm{i}} \quad$ is the improvement in $\left(\mathrm{q}_{\mathrm{i}}\right)$ when the stock level (n) of component $\mathrm{i}$ is raised from

$$
S i=n \text { to } S i=n+i \text {; }
$$

$\left(q_{i}\right)_{n} \quad$ is the item operational availability when $\mathrm{S}_{\mathrm{i}}=n$

$\left(q_{i}\right)_{n+i}$ is the item operational availability when $S_{i}=n+i$.

The formula gives the availability contribution of an item $i$ by increasing it by one spare component.

When the improvement factor is divided by the unit price $\mathrm{C}_{\mathrm{i}}$ of component $\mathrm{i}$, the required sorting factor $\left(S F_{i}\right)$ is obtained:

$$
S F_{i}=\frac{I_{i}}{C_{i}}
$$


Proceeding of $4^{\text {th }}$ International Conference on Engineering Mathematics and Physics

S-EM III 1166

Items of higher $S F_{i}$ will be introduced first in the recommended spare list for procurement action. Then the new item availability $q_{i}$ is calculated again based on the new stock level of item $i$. The availability contribution is determined, and will be introduced into the aircraft system availability $\left(\mathrm{A}_{0}\right)$ as follows:

$$
\left(A_{o}\right)_{\text {New }}=\left(A_{o}\right)_{\text {Old }} \cdot \frac{\left(q_{i}\right)_{\text {New }}}{\left(q_{i}\right)_{\text {Old }}}
$$

\subsection{Optimization Algorithm}

A. Define the aircraft utilization requirements in operational hours/aircraft/month;

B. Calculate the initial value of the aircraft $A_{0}$ using the parameter of the initial stock level of aircraft components (i.e. $\mathrm{S}_{\mathrm{i}}, \mathrm{MTBD}_{\mathrm{i}}, \mathrm{RTS}_{\mathrm{i}}, \mathrm{NRTS}_{\mathrm{i}}, \mathrm{TTRI}$, TTRD, and QPA). This is carried out by the application of equations 1 through 8 ;

C. If the resulted operational availability is not satisfactory increase the stock level of all considered components by 1 each, then;

D. Calculate the improvement factor, equation 9 for each component after the first stock level increase;

E. Calculate the sort value for each component using equation 10, then;

F. Arrange the components in ascending order according to the sort value;

G. Calculate the new $\mathrm{A}_{0}$, using equation 11, and the accumulated cost each time;

$\mathrm{H}$. Repeat steps $\mathrm{C}$ through $\mathrm{G}$ until the required $\mathrm{A}_{0}$ is achieved or the available budget is exhausted.

\section{TYPICAL EXAMPLE}

In this work, a typical example is introduced and the problem is defined and analyzed. An aircraft system of 40 aircraft is procured and deployed into 2 squadrons in one operational site according to the operational and maintenance requirements. The aircraft system availability is low. Also, system-operating costs may be high and the required utilization is difficult to investigate. This may decrease the aircraft system readiness and thus threaten the national security. Therefore the goal is to arise the availability value by procurement of spares according to an available budget.

\section{PROBLEM SOLVING APPROACH}

The following steps are to establish the required problem solving:

1. Using the design, operations and support factors and the item data required as shown in tables 1 and 2 .

2. Utilizing the aircraft operational availability, $\mathrm{A}_{0}$, as an important external indicator to predict the performance of the aircraft system.

3. Using the A AM to solve this problem.

Table 1. Operational data

\begin{tabular}{|c|c|}
\hline No of Aircraft (NAC) & 40 Aircraft \\
\hline Utilization Requirements (UTR) & 15 Hours \\
\hline Flying Days per Month (FDM) & 20 Days \\
\hline
\end{tabular}


Table 2. Spare item data

\begin{tabular}{|c|c|c|c|c|c|c|c|c|}
\hline No & Type & S & QPA & MTBD & NRTS & TTRI & TTRD & COST \\
\hline 1 & 74ACO & 18 & 1 & 325 & 0.86 & 15 & 180 & 10000 \\
\hline 2 & 74ANO & 7 & 1 & 300 & 0.04 & 15 & 180 & 2000 \\
\hline 3 & 74 ADO & 7 & 1 & 100 & 0.24 & 15 & 180 & 6000 \\
\hline 4 & 74 AMO & 9 & 1 & 1250 & 0.53 & 15 & 180 & 5000 \\
\hline 5 & $74 \mathrm{DFO}$ & 9 & 1 & 230 & 0.73 & 15 & 180 & 7000 \\
\hline
\end{tabular}

\section{RESULTS AND CONCLUSIONS}

Using a given initial aircraft system information, the AAM has been applied. The data required are taken from tables 1 and 2. The results are presented in tables 3 and 4 . As we see, the availability of the system was very low $\left(\mathrm{A}_{0}=0.683\right)$. After the optimization, it is very high $\left(\mathrm{A}_{0}=\right.$ $0.999)$ through an available budget of $160,000 \$$. The gain is $1 \%$ of $\mathrm{A}_{0}$ per $5063.5 \$$ paid. The stock level is raised to a new level as shown in table 3 . The spare items are chosen so that they give a big availability improvement against their costs.

Table 3. Results of the optimization

\begin{tabular}{|c|c|c|c|c|}
\hline No & Type & Initial Stock Level & New Stock Level & Items Added \\
\hline 1 & $74 \mathrm{ACO}$ & 18 & 24 & 6 \\
\hline 2 & $74 \mathrm{ANO}$ & 7 & 7 & 6 \\
\hline 3 & $74 \mathrm{ADO}$ & 7 & 29 & 22 \\
\hline 4 & $74 \mathrm{AMO}$ & 9 & 9 & 0 \\
\hline 5 & $74 \mathrm{DFO}$ & 9 & 23 & 14 \\
\hline
\end{tabular}

Table 4. Results of the optimization

\begin{tabular}{|c|c|c|}
\hline & Before Optimization & After Optimization \\
\hline $\mathrm{A}_{o}$ & 0.683 & 0.999 \\
\hline Budget (\$) & - & 160000 \\
\hline Gain & - & $5063.5 \$ / 1 \% \mathrm{~A}_{o}$ \\
\hline
\end{tabular}

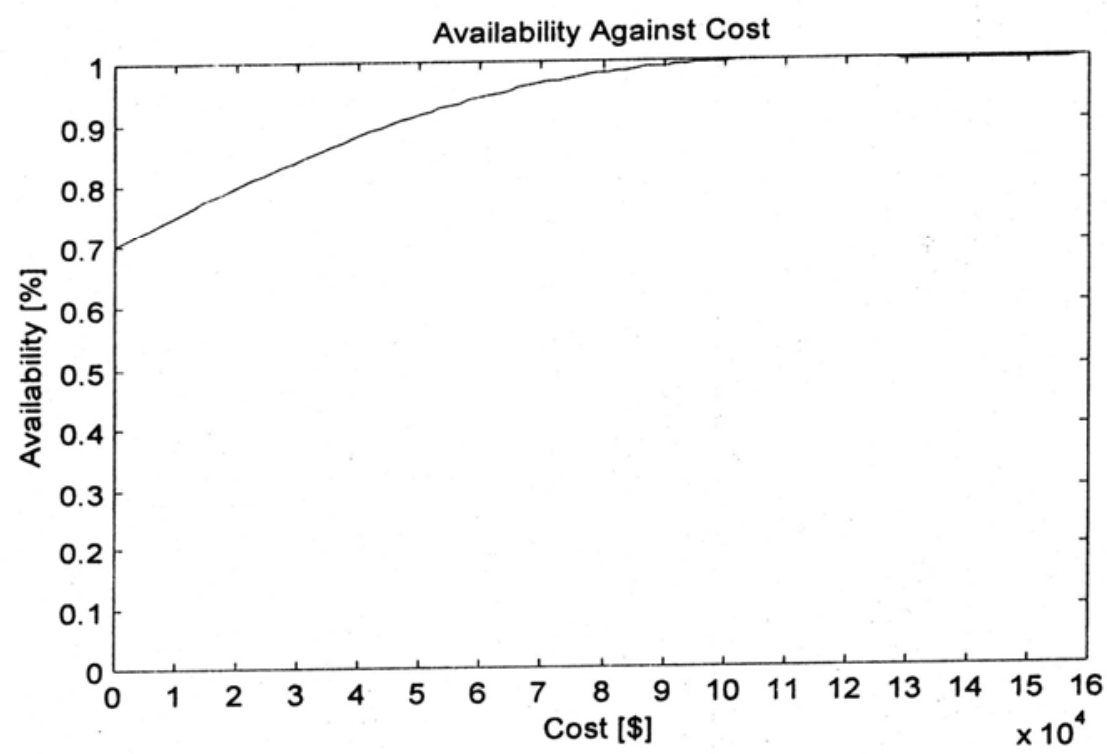

Fig. 3. Availability against the budget 
Proceeding of $4^{\text {th }}$ International Conference on Engineering Mathematics and Physics

Figure 3 represents the availability against the budget. It is clear from the curve slope that the rate of change at first greater compared with the last steps of the curve against the costs, therefore the user can see very clear the proper budget which can give the satisfied $\mathrm{A}_{0}$. In our example, a required budget to give an availability of $A o=0.99$, is $100,000 \$$. From the analysis of the previous example, we can conclude that:

1.The objective of the work is to minimize the expected back order, by another words, maximize the operational availability.

2. The spare availability calculation technique provides accurate computation of the spares necessary to meet an availability requirement.

\section{REFERENCES}

[1] Atef A. Ayed, "Analysis of Aircraft Logistic Support Using a Life Cycle Cost Approach," M.Sc. Thesis, Military Technical College, 1997.

[2] Mohamed M. Elmaadawy and Atef O. Sherif, "Aircraft Acquisition and Operation in a Total Quality Environment," Proceedings of the SOLE, $31^{\text {st }}$ Annual Symposium, 1996.

[3] William T. Baylis, "An Analysis of the Impact of System Support on Cost,". Proceedings of the SOLE, $34^{\text {th }}$ Annual Symposium, 1999. 